\title{
Betahistin in einer großen Studie bei Morbus Menière nicht wirksamer als Placebo
}

Fragestellung: Bietet das Antivertiginosum Betahistin eine wirksame Prophylaxe von Schwindelattacken, die im Rahmen eines Morbus Menière auftreten?

Hintergrund: Der Morbus Menière ist die dritthäufigste Ursache von Schwindelattacken nach dem paroxysmalen Lage- und Lagerungsschwindel und dem phobischen Schwankschwindel. Während der Attacken, die plötzlich einsetzen und über mehrere Stunden anhalten, kommt es neben dem Drehschwindel und den entsprechenden vegetativen Begleiterscheinungen auch $\mathrm{zu}$ einer Hörminderung auf dem betroffenen Ohr, einem Tinnitus und einem Druckgefühl. Betahistin ist seit vielen Jahren zur Prophylaxe von Attacken der Menièrschen Erkrankung zugelassen. Das Deutsche Schwindelzentrum in München hat nun zum ersten Mal eine große placebokontrollierte Studie zur Wirksamkeit von Betahistin bei der Menièrschen Erkrankung durchgeführt, die vom Bundesministerium für Bildung und Forschung (BMBF) unterstützt wurde.

Adrion C, Fischer CS, Wagner J et al. Efficacy and safety of betahistine treatment in patients with Meniere's disease: primary results of a long term, multicentre, double blind, randomised, placebo controlled, dose defining trial (BEMED trial). BMJ 2016; 352: h6816
Patienten und Methodik: Bei der hier vorliegenden Untersuchung handelte es sich um eine doppelblinde, randomisierte und placebokontrollierte Multicenterstudie, die in 14 deutschen Schwindelzentren durchgeführt wurde. Für die Studie wurden zwischen März 2008 und No- vember 2012 erwachsene Patienten mit der Diagnose eines unioder bilateralen Morbus Menière rekrutiert. Die Studienteilnehmer erhielten entweder Placebo, niedrig dosiertes Betahistin $(2 \times$ $24 \mathrm{mg} / \mathrm{Tag}$ ) oder eine hohe Dosis Betahistin ( $3 \times 48 \mathrm{mg} / \mathrm{Tag})$. Der primäre Endpunkt war die Zahl der Menière-Attacken in einem Zeitraum von 30 Tagen verglichen mit der Baselineperiode in den letzten drei Monaten der Studie (Monat 7 bis 9). Sekundäre Studienendpunkte waren die Dauer und die Schwere der Attacken sowie die Lebensqualität der Patienten.

Ergebnisse: In die Studie wurden insgesamt 221 Patienten aufgenommen. Für die Endauswertung standen 72 Patienten in der Placebo-, 70 in der Niedrigdosis-und 72 in der Hochdosisbehandlungsgruppe zur Verfügung. Die Studienteilnehmer waren im Schnitt 55 Jahre alt. Die Anzahl der Menière-Attacken vor Einschluss in die Studie wurde nicht dokumentiert.

Während der Studie kam es zu einem signifikanten Rückgang der Anzahl der Menière-Attacken in allen drei Behandlungsgruppen. Die mittlere Anzahl der Menière-Attacken während der Studie lag zwischen 2,7 und 3,2. Auch für alle untersuchten sekundären Endpunkte ergaben sich keine Unterschiede. Die Behandlung mit Betahistin wurde insgesamt hervorragend vertragen.

Schlussfolgerung: Die Behandlung mit niedrig oder hoch dosiertem Betahistin hat im Vergleich zu Placebo keinen signifikanten Nutzen im Hinblick auf die Reduktion der Häufigkeit von Menière-Attacken.

\section{Bei Morbus Menière weiterhin mit hoch dosiertem Betahistin behandeln}

Die hier referierte Studie ist bereits im Februar 2016 erschienen, wurde vom Referenten aber leider zu diesem Zeitpunkt übersehen. Das Ergebnis der Studie ist extrem überraschend. Jeder Kliniker, der mit Schwindelpatienten arbeitet, hatte bisher den Eindruck, dass Betahistin, insbesondere in der hohen Dosis, eine gute prophylaktische Wirkung beim Morbus Menière hat. Dies fiel insbesondere dann auf, wenn Patienten eine signifikante Reduktion ihrer Menière-Attacken unter Therapie erfuhren und nach Beendigung der Therapie die Zahl der Menière-Attacken wieder anstieg. Über das negative Ergebnis der Studie kann nur spekuliert werden. Eine Möglichkeit ist, dass es einen extrem hohen Placeboeffekt gab, was unter anderem dadurch erklärt werden kann, dass bei diesen Patienten sehr viele Untersuchungen durchgeführt wurden. Eine weitere Möglichkeit ist, dass ein Teil der Patienten gar nicht unter Morbus Menière litt, sondern eine vestibuläre Migräne hatte.
Die Auswirkungen für den klinischen Alltag sind schwer zu beurteilen. Der Referent würde weiterhin bei Patienten mit häufigen und beeinträchtigenden Menière-Attacken dazu raten, diese Patienten mit hoch dosiertem Betahistin zu behandeln.

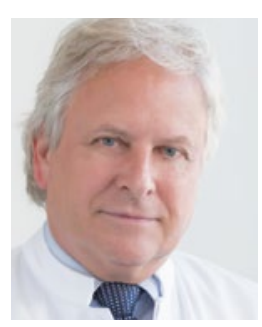

Prof. Dr. med. Hans-Christoph Diener, Essen

Seniorprofessur für klinische

Neurowissenschaften,

Universitätsklinikum Essen

E-Mail:h.diener@uni-essen.de 